\title{
Surgical Decision Making in Oral Mucosal Melanoma with Lymphadenopathy: Review of Literature
}

\author{
${ }^{1}$ Niladri Bakshi, ${ }^{2}$ Srijon Mukherjee, ${ }^{3}$ Yogendra Singh Chauhan
}

\begin{abstract}
The oral mucosal melanoma (OMM) comprises less than $1 \%$ of all malignant melanomas. Most mucosal melanomas occur in occult site, which together with lack of early and specific signs contribute to late and poor diagnosis. Because of their rareness, the knowledge about their pathogenesis and risk factors is insufficient, and also there are not well-established protocols for staging and treatment of mucosal melanomas with lymphadenopathy. Surgery is the mainstay of treatment. Radiotherapy and other adjunctive therapies can provide better local control in some locations, but did not show improvement in survival. In this article, we present a case of 52-year-old female with OMM (excision performed elsewhere) presenting with large left upper cervical swelling after 2 years post-excision. We will review the literature that help us in taking decision in the management of OMMs in light of following patient.
\end{abstract}

Keywords: Oral mucosal melanoma, Surgery, Upper neck swelling, Radiotherapy.

How to cite this article: Bakshi N, Mukherjee S, Chauhan YS. Surgical Decision Making in Oral Mucosal Melanoma with Lymphadenopathy: Review of Literature. Int J Head Neck Surg 2014;5(3):135-139.

\section{Source of support: Nil}

Conflict of interest: None

\section{INTRODUCTION}

Primary oral mucosal melanomas (OMMs) are far less common than their cutaneous counterparts. The incidence of OMMs varies between 0.2 and $0.8 \%$ of all melanomas in Europe and US. ${ }^{1,2}$ In the US, OMMs account for $<1 \%$ of all melanomas, with an incidence of OMM being 1.2 cases per 10 million people per year. ${ }^{3}$ However, OMMs made up $7.5 \%$ of all melanomas and constituted $34.4 \%$ of all mucosal melanomas in Japan. ${ }^{2}$

\footnotetext{
${ }^{1}$ Associate Fellow, ${ }^{2}$ Chief Consultant, ${ }^{3}$ Surgical Fellow

1,2Department of Oral and Maxillofacial Surgery, Calcutta Institute of Maxillofacial Surgery and Research, Kolkata West Bengal, India

${ }^{3}$ Department of ENT, Head and Neck Surgery, Calcutta Institute of Maxillofacial Surgery and Research, Kolkata West Bengal, India

Corresponding Author: Yogendra Singh Chauhan, Surgical Fellow, Department of ENT, Head and Neck Surgery, Calcutta Institute of Maxillofacial Surgery and Research, Kolkata, West Bengal, India Phone: 08017127605, e-mail: dr.yogendrared@ yahoo.com
}

OMMs often occur in patients from 40 to 70 years of age with mean of 53 years. ${ }^{2}$ The most frequent site are palate and maxillary gingival, accounting for more than $50 \%$ of all OMMs. ${ }^{3}$ Mucosal melanomas tend to present at a higher stage, and are more aggressive and in a vertical growth phase of the disease. ${ }^{3}$ Therefore, the prognosis of OMM is very poor, and 5-year survival rate for OMMs is very low, ranging from 13 to $22 \%{ }^{1,4}$

Because of the less-common occurrence of OMMs, tumor progression, biologic behavior, treatment modalities, prognosis of OMMs are usually obtained from case reports and small series studies. The purpose of present article is to report and review a post excision oral mucosal melanoma presenting with large left upper cervical neck swelling and to consider the facts to decide when to perform elective neck dissection.

\section{CASE REPORT}

A 52-year-old female presented to our institute with large left upper cervical painless neck mass which was gradually progressive to reach a present size in 9 months duration. History disclosed that she had an excision of pigmented nodular lesion from retromolar area 2 years back (Fig. 1). Histopathological examination of nodular lesion revealed to be a malignant mucosal melanoma (Figs 2 and 3). Wait and watch policy was considered by attending surgeon. Intraoral examination was noncontributory. On neck examination, $12 \times 10 \mathrm{~cm}$ upper cervical lymph nodal mass which was nontender, local temperature was not raised, hard, mobile with no fixity to underlying musculature or skin (Fig. 4). Fine needle aspiration cytology (FNAC) report suggested malignant melanoma. A whole body contrast-enhanced computed tomography (CECT) scan was performed to know the extranodal metastasis all over the body which showed no clinicoradiological abnormality. Patient was posted to undergo radical neck dissection clearing up level I, II, III, IV, V, sternocleidomastoid, internal jugular vein, spinal accessory nerve along with submandibular gland. The specimen was removed in toto. Gross specimen examination showed $11 \times 9 \times 5.5 \mathrm{~cm}$ with salivary gland $4 \times 2.5 \times 2 \mathrm{~cm}$ with 41 lymph node with largest of which markedly pigmented and $6 \mathrm{~cm}$ diameter in level II. Histopathological examination revealed tumor metastasis in 
06/65 at level II extranodal involvement of soft tissue and infiltration of tumor in salivary gland. Muscle and vessels are free (Fig. 5). Examination was suggestive of malignant melanoma.

\section{DISCUSSION}

The mucosal membranes are rare sites for primary malignant melanoma. The presence of melanocytes in the mucosal membrane of respiratory, urogenital and alimentary tract explains the occurrence of malignant melanoma in these sites. The initial symptom and sign of OMMs is often a pigmented growth or swelling. The surface may be smooth, with an intact or ulcerated overlying mucosa. The color may uniformly brown or black or may show variation of color, with black, brown, gray, purple and red shades or depigmentation. ${ }^{5}$

Other presenting signs and symptoms includebleeding, ill-fitting dentures, pain, increased mobility of teeth and delayed healing of extraction sockets. OMM is more aggressive and abundant blood supply of the oral cavity may permit blood vessel invasion and hematogenous dissemination early in course of the disease. ${ }^{6}$ Regional lymphadenopathy may be present and connotes a poor prognosis. ${ }^{7}$

When oral pigmentation cannot be confidently diagnosed as benign on clinical grounds, a biopsy is mandatory. An excisional biopsy with a 1 to $2 \mathrm{~mm}$ margin for small lesion or an incisional biopsy through the thickest or the most suspicious part of the tumor in case of large lesion is required. ${ }^{8}$ Fine needle aspiration or exfoliative cytology of primary pigmented lesion is contraindicated. It has been suggested that cutting into malignant neoplasm during an incisional biopsy or other invasive procedure could result in accidental dissemination of malignant cells within the adjacent tissues or even in the blood or lymphatic stream, with the subsequent risk of local recurrences or regional or distant metastasis. ${ }^{9}$

Oral mucosal melanoma can be histologically subclassified into: (1) in situ melanoma, which is limited to the limited the epithelium and epithelial connective tissue interface, (2) melanoma with invasive pattern, in which the neoplasm extends into connective tissue and (3) melanoma with combined pattern of invasive melanoma with in situ component. ${ }^{5}$

A simple tumor node metastasis (TNM) clinical staging, recognizing three stages, has shown to be of prognostic value as follows: ${ }^{9-11}$

- Stage I: Primary tumor presents only (any T N0M0)

- Level I: Pure in situ melanoma without evidence of invasion or in situ melanoma with 'microinvasion'

- Level II: Invasion up to lamina propria

- Level III: Deep skeletal tissue invasion into skeletal muscle, bone or cartilage
- Stage II: Tumor metastatic to regional lymph nodes (any T N1M0)

- Stage III: Tumor metastatic to distant sites (any T, any NM1)

\section{SURGICAL DECISION IN PRIMARY OMMS WITH LYMPHADENOPATHY}

Surgery remains the treatment of choice for OMMs, although the outcome is still disappointing. ${ }^{1,12}{\text { Tanaka et } \mathrm{al}^{8}}^{8}$ reviewed 140 OMM patients in Japan, among whom $116(72.5 \%)$ received surgical intervention and only $17(14.7 \%)$ lived for more than 3 years. In this study, one patient received surgical excision of the primary OMM with modified radical dissection and another only underwent surgical resection of primary tumor. The former survived 25 months and latter lived 14 months after initial treatment. Malignant melanoma is traditionally thought to be radioresistant; therefore, radiotherapy is usually used as an adjuvant treatment after surgery or as a primary modality for the elderly and medically compromised patients. ${ }^{6,13}$ Gorsky and Epstein ${ }^{14}$ reported a longer survival time (54 months) in patients receiving

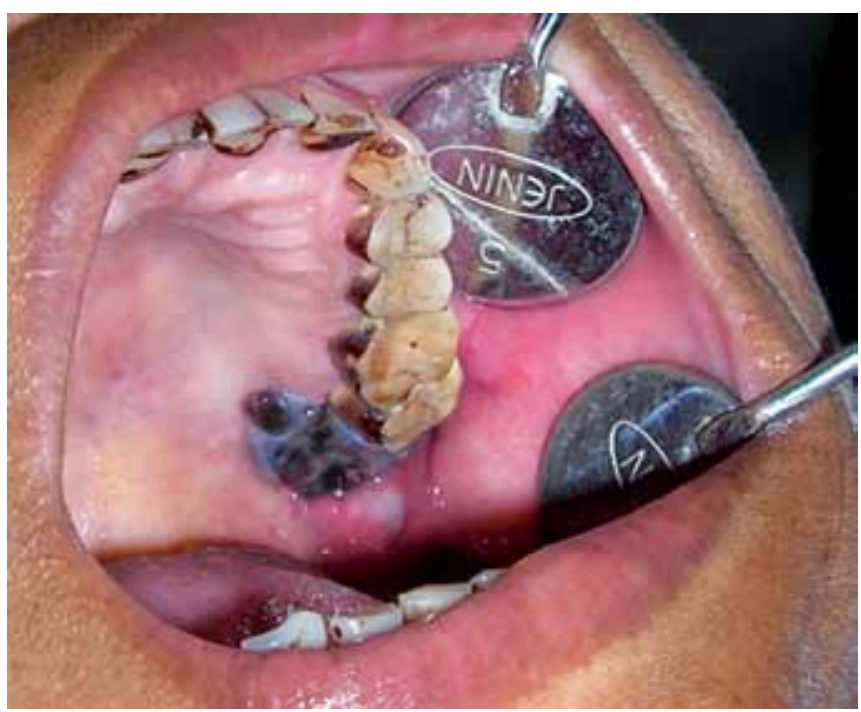

Fig. 1: Typical appearance of oral mucosal melanoma

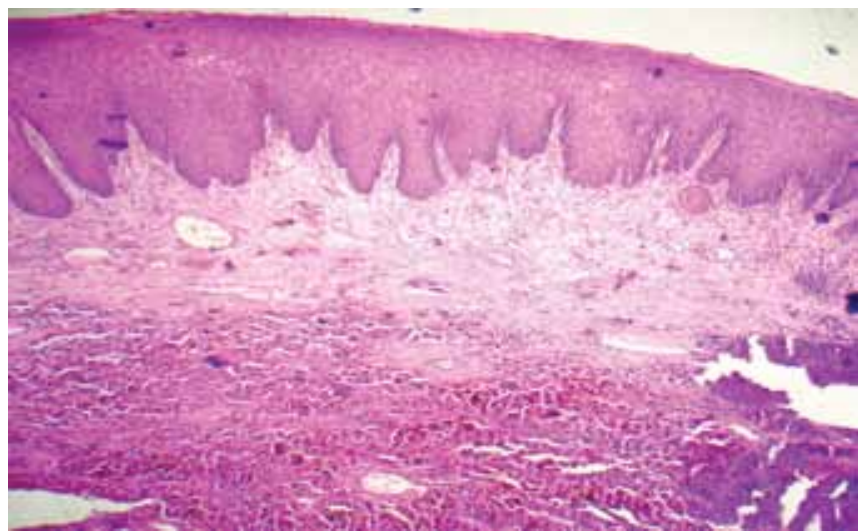

Fig. 2: Low magnification showing melanocytes 
surgery and radiation than in patients receiving radiation alone (5 months).

Principles defined for treatment of all melanomas should be performed by wide resection. Surgical margins also depend on the thickness of the tumor. In patients with tumors less than $1 \mathrm{~mm}$ thickness, a surgical margin of $1 \mathrm{~cm}$ has been shown to be adequate, compared with wide tumor margin resection of $3 \mathrm{~cm} .{ }^{15}$ None of these patients developed local recurrences

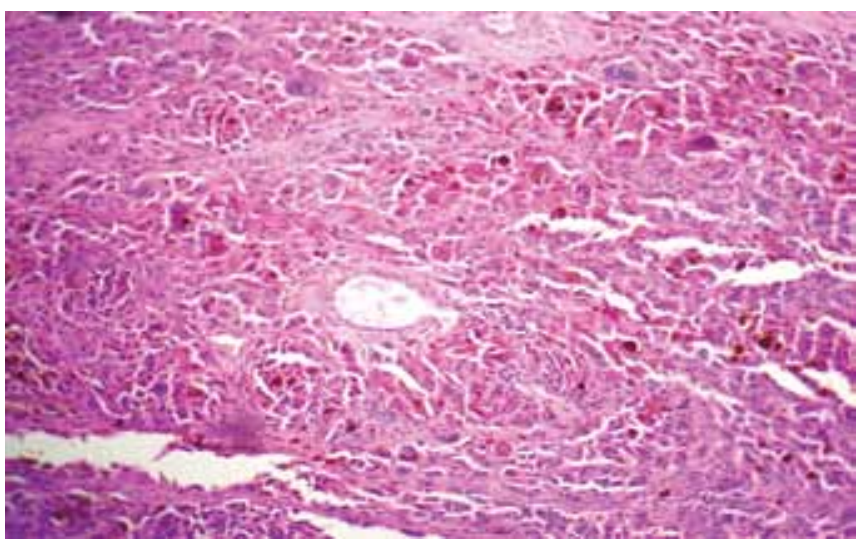

Fig. 3: High magnification showing melanocytes

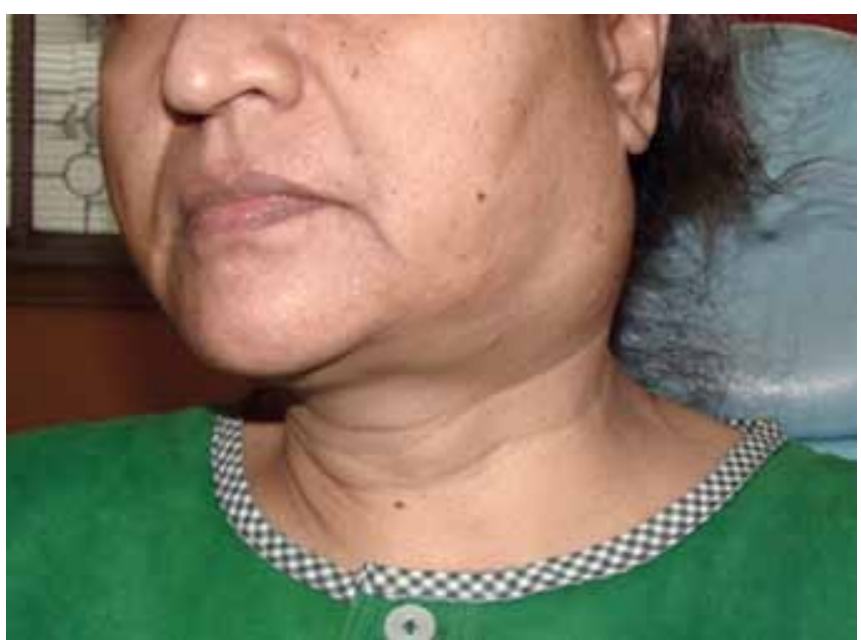

Fig. 4: Same patient with large level II neck node

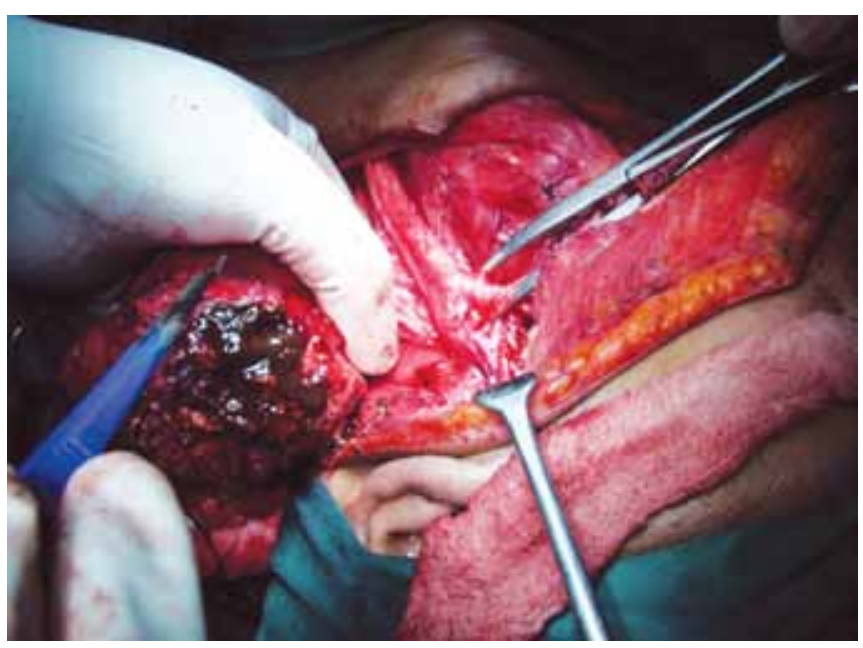

Fig. 5: Large melanomatous mass in the follow-up period revealing an adequate resection extent. In patients with tumor thickness of 1 to $2 \mathrm{~mm}$ local recurrences were found in both study groups of patients receiving resection margins of 1 or $3 \mathrm{~cm}$ without significant differences, although more were found in the $1 \mathrm{~cm}$ group. ${ }^{15}$ The evidence that resection of 1 to $2 \mathrm{~cm}$ around the tumor are sufficient and show similar results in local control and overall survival. ${ }^{16,17}$ The surgical resection within oral cavity remains problematic because wide resection margins require also reconstruction technique after tumor ablation to avoid mutilation, functional impairment and poor quality of life. ${ }^{18-20}$ Regional lymph nodes are the most common sites of metastases for all melanomas. Palpable lymph nodes in neck or fixed to the adjacent tissues should be suspicious for metastases. Radiologically, lymph nodes larger than $1 \mathrm{~cm}$ must also be considered to be involved by metastases. There exists a correlation between the tumor thickness and the occurrence of regional lymph node metastases. Primary lesions less than $1 \mathrm{~mm}$ thickness are considered to yield a rate of $<10 \%$ lymph node metastases, 1.01 to $2.00 \mathrm{~mm}$ are accounting for about $20 \%, 2.01$ to $4.00 \mathrm{~mm}$ are accounting for $33 \%$ and $>4.00 \mathrm{~mm}$ are associated with a risk of lymph node involvement of more than $40 \%$ at the time of staging. ${ }^{21}$ Another study evaluated an exponential increasing of lymph node metastases if the tumor becomes thicker. A thickness of 0.76 to $1.50 \mathrm{~mm}$ was associated with regional lymph node metastases in 2 to $25 \%$ of cases. In tumors with a thickness between 1.51 and $4.00 \mathrm{~mm}$, the rate of regional lymph node developed to $57 \%$. A tumor thickness larger than $4 \mathrm{~mm}$ was associated with microscopic presence of metastases within lymph nodes. ${ }^{22}$ There has been evidence that lymph node dissection resulted in an increase of overall survival compared to patients receiving palliative treatment to the neck only. ${ }^{22}$ Negative lymph nodes are strong prognostic factor for survival, whereas lymph node metastases yield a 6 times higher relative risk for death. ${ }^{23}$

Patients who suffered from oral mucosal melanomas are often diagnosed at an advanced stage followed by ulceration, microsatellites or regional nodal metastases. ${ }^{10,11,18}$ This high rate of regional lymph node metastases means that patients at risk should considered for therapeutic elective neck dissection with a low threshold for surgery. Although no randomized trials on the treatment of the regional lymph nodes for oral mucosal malignant melanoma exist due to its rarity of this tumor, there is little doubt about this treatment approach. ${ }^{18,23}$ As the OMMs are very aggressive and the texture of mucosa is different from the cutis with an earlier occurrence of lymph node metastases, there is actually no role of sentinel lymph node technique, as the risk of 
Flow Chart 1: Oral mucosal melanoma management protocol

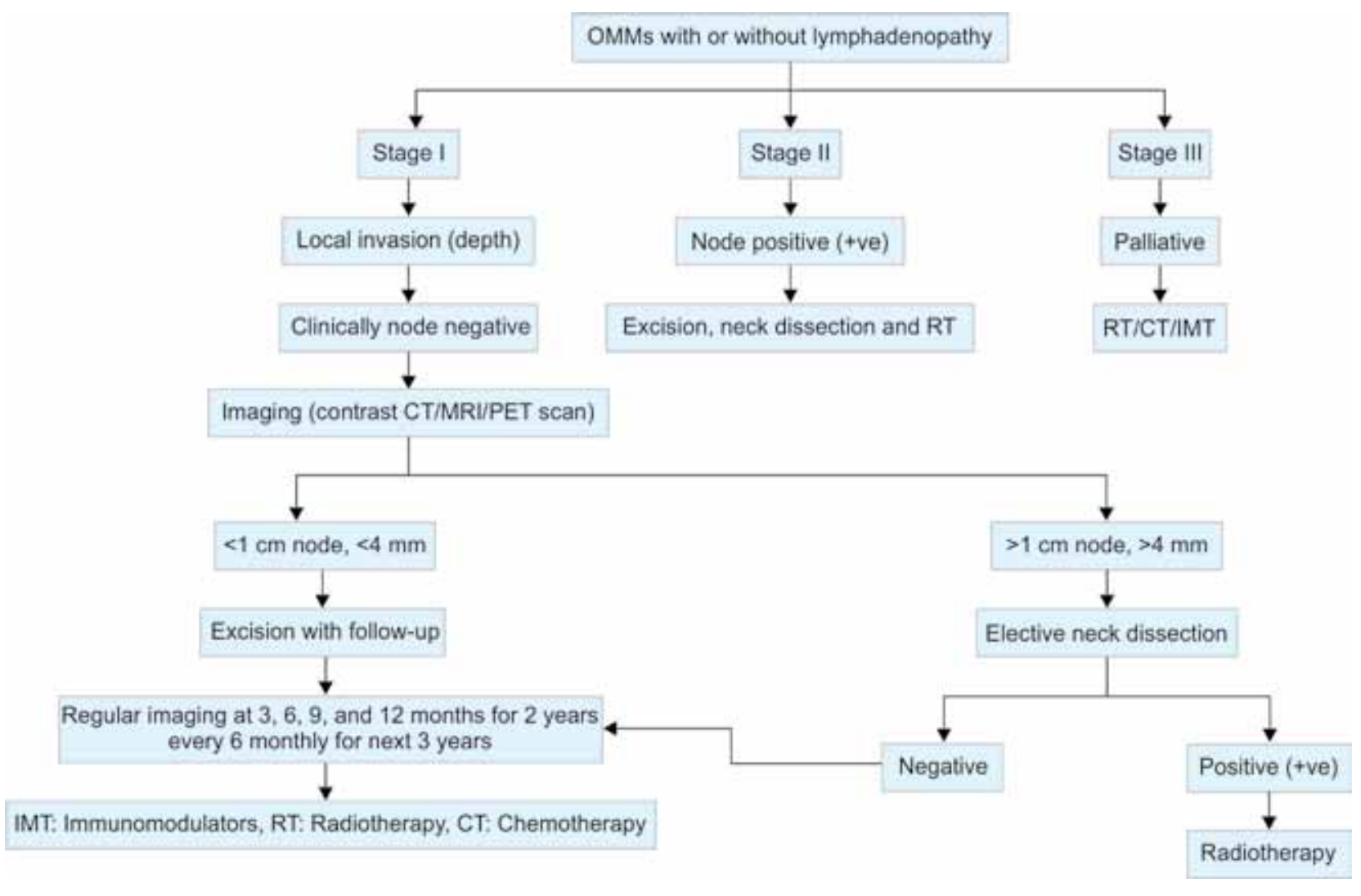

micrometastases, has to be excluded by standard therapeutic neck dissection. ${ }^{10,12}$

\section{ROLE OF ADJUVANT THERAPIES BESIDES SURGERY}

The most established therapies besides surgery are radiotherapy and cytotoxic chemotherapy. Radiotherapy has been used to control local recurrence. ${ }^{16}$ Studies have not shown increase in survival rate with cytotoxic therapies.

\section{TREATMENT PROTOCOL FOR OMMS}

On the basis of literature reviewed, we would like to suggest our protocol for the treatment of OMMs with or without lymphadenopathy to help in surgical decision making:

Relation between depth of invasion and lymphadenopathy $^{21}$ :

- <1 mm: $10 \%$

- $1.01-2.00 \mathrm{~mm}: 20 \%$

- $2.01-4.00 \mathrm{~mm}: 33 \%$

- $>4.00 \mathrm{~mm}: 40 \%$

\section{INDICATIONS OF NECK DISSECTION}

- Stage I with depth of invasion $<4 \mathrm{~mm}$, clinically node negative, imaging node $>1 \mathrm{~cm}$ (Flow Chart 1 )

- Stage I with depth of invasion $>4 \mathrm{~mm}$, clinically node negative, imaging node $<1 \mathrm{~cm}$

- Stage II node positive.

\section{CONCLUSION}

The incidence of oral melanoma is very low, and results of the treatment are still poor partly due to the advanced stage of tumor at presentation. No single management strategy or guideline can be considered the standard of care on the basis of current data. To date, due to the rarity of this tumor entity, no randomized trials exist demonstrating any optimal treatment algorithm.

The tumor thickness is a variable that most accurately determines therapy and prognosis; therefore, the extent of surgery margins should be decided based on the invasive depth of the primary lesion, the neck addressed on the basis of imaging staging. The suggestive treatment protocol by us can be helpful in surgical decision making and proceed in stepwise manner for the management of oral mucosal melanomas.

\section{REFERENCES}

1. Rapini RP, Golitz LE, Greer RO Jr, Krekorian EA, Poulson T. Primary malignant melanoma of the oral cavity: a review of 177 cases. Cancer 1985;55:1543-1551.

2. Takagi M, Ishikawa G, Mori W. Primary malignant melanoma of the oral cavity in Japan: with special reference to mucosal melanosis. Cancer 1974;34:358-370.

3. Hicks MJ, Flaitz CM. Oral mucosal melanoma: epidemiology and pathobiology. Oral Oncol 2000;36:152-169.

4. Trodahl JN, Sprague WG. Benign and malignant melanocytic lesions of the oral mucosa: an analysis of 135 cases. Cancer 1970;25:812-823. 
5. Barker BF, Carpenter WM, Daniels TE, et al. Oral mucosal melanomas: the westop banff workshop proceedings. Oral Surg Oral Medi Oral Pathol Oral Radiol Endodontics 1997;83(6):672-679.

6. Graniel LCM, Carrillo OFJ, Garcia MA. Malignant melanoma of the oral cavity: diagnosis and treatment experience in Mexican population. Oral Oncol 1999;35:425-430.

7. Van der Waal L, Van der Kwast WAM. Malignant melanoma. Oral Pathology. Quintessence, Chicago, USA; 1988.

8. Tanaka N, Amagasa T, Iwaki H, et al. Oral malignant melanoma in Japan. Oral Surg Oral Med Oral Pathol 1994;78(1): 81-90.

9. Meleti M, Leemans R, Mooi WJ, Vescovi P, Van der waal L. Oral malignant melanoma: a review of literature. Oral Oncol 2007;43(2):116-121.

10. Prasad ML, Patel SG, Huvos AG, Shah JP, Busam KJ. Primary mucosal melanoma of the head and neck: a proposal for microstaging localized, stage I (lymph node negative) tumors. Cancer 2004;100(8):1657-1664.

11. Patel SG, Prasad ML, Escrig M, et al. Primary mucosal malignant melanoma of the head and neck. Head and Neck 2002; 24(3):247-257.

12. Umeda M, Shimada K. Primary malignant melanoma of the oral cavity: its histological classification and treatment. Br J Oral Maxillofac Sur 1994;32:39-47.

13. McCaffery TV, Neel HB, Gaffey TA. Malignant melanoma of the oral cavity: review of 10 cases. Laryngoscope 1980; 90:1329-1335.

14. Gorsky M, Epstein JB. Melanoma arising from the mucosal surfaces of the head and neck. Oral Surg Oral Med Oral Pathol Oral Radiol Endod 1998;86:715-719.

15. Veronesi U, Cascinelli N, Adamus J, Balch C, Bandiera D, Barchuk A, Bufalino R, Craig P, De Marsillac J, Durand JC, et al. Thin stage I primary cutaneous malignant melanoma. Comparison of excision with margins of 1 or $3 \mathrm{~cm}$. N Engl J Med 1988;318:1159-1162.
16. Balch CM, Urist MM, Karakousis CP, Smith TJ, Temple WJ, Drzewiecki K, Jewell WR, Bartolucci AA, Mihm MC Jr, Barnhill R, et al. Efficacy of $2 \mathrm{~cm}$ surgical margins for intermediate-thickness melanomas (1 to $4 \mathrm{~mm}$ ). Results of a multi-institutional randomized surgical trial. Ann Surg 1993;218:262-267; discussion 267-269.

17. Heaton KM, Sussman JJ, Gershenwald JE, Lee JE, Reintgen DS, Mansfield PF, Ross MI. Surgical margins and prognostic factors in patients with thick $(>4 \mathrm{~mm})$ primary melanoma. Ann Surg Oncol 1998;5:322-328.

18. Mücke T, Hölzle F, Kesting MR, Loeffelbein DJ, Robitzky LK, Hohlweg-Majert B, Tannapfel A, Wolff KD. Tumor size and depth in primary malignant melanoma in the oral cavity influences survival. J Oral Maxillofac Surg 2009;67:1409-1415.

19. Mücke T, Hölzle F, Wagenpfeil S, Wolff KD, Kesting M. The role of tumor invasion into the mandible of oral squamous cell carcinoma. J Cancer Res Clin Oncol 2011;137:165-171.

20. Mücke T, Wolff KD, Wagenpfeil S, Mitchell DA, Hölzle F. Immediate microsurgical reconstruction after tumor ablation predicts survival among patients with head and neck carcinoma. Ann Surg Oncol 2010;17:287-295.

21. Morton DL, Cochran AJ, Thompson JF, Elashoff R, Essner R, Glass EC, Mozzillo N, Nieweg OE, Roses DF, Hoekstra HJ, et al. Sentinel node biopsy for early-stage melanoma: accuracy and morbidity in MSLT-I, an international multicenter trial. Ann Surg 2005;242:302-311; discussion 311-303.

22. Balch CM, Soong S, Ross MI, Urist MM, Karakousis CP, Temple WJ, Mihm MC, Barnhill RL, Jewell WR, Wanebo HJ, et al. Long-term results of a multi-institutional randomized trial comparing prognostic factors and surgical results for intermediate thickness melanomas (1.0 to $4.0 \mathrm{~mm}$ ). Intergroup Melanoma Surgical Trial. Ann Surg Oncol 2000;7:87-97.

23. Balch CM, Morton DL, Gershenwald JE, McMasters KM, Nieweg OE, Powell B, Ross MI, Sondak VK, Thompson JF. Sentinel node biopsy and standard of care for melanoma. J Am Acad Dermatol 2009;60:872-875. 